\title{
Correction to: Advances in Ocular Imaging in Glaucoma
}

Rohit Varma, Benjamin Y. Xu, Grace M. Richter, and Alena Reznik

\section{Correction to:}

R. Varma et al. (eds.), Advances in Ocular Imaging in Glaucoma, Essentials in Ophthalmology, https://doi.org/10.1007/978-3-030-43847-0

The book was inadvertently published with incorrect affiliation of Rohit Varma in copyright page. His affiliation has now been amended to "Southern California Eye Institute, CHA Hollywood Presbyterian Medical Center, Los Angeles, CA, USA". 